\title{
Sparse recovery under weak moment assumptions
}

\author{
Guillaume Lecué ${ }^{1,3} \quad$ Shahar Mendelson ${ }^{2,4,5}$
}

March 4, 2015

\begin{abstract}
We prove that iid random vectors that satisfy a rather weak moment assumption can be used as measurement vectors in Compressed Sensing, and the number of measurements required for exact reconstruction is the same as the best possible estimate - exhibited by a random Gaussian matrix. We then show that this moment condition is necessary, up to a $\log \log$ factor. In addition, we explore the Compatibility Condition and the Restricted Eigenvalue Condition in the noisy setup, as well as properties of neighbourly random polytopes.
\end{abstract}

\section{Introduction and main results}

Data acquisition is an important task in diverse fields such as mobile communications, medical imaging, radar detection and others, making the design of efficient data acquisition processes a problem of obvious significance.

The core issue in data acquisition is retaining all the valuable information at one's disposal, while keeping the 'acquisition cost' as low as possible. And while there are several ways of defining that cost, depending on the problem (storage, time, financial cost, etc.), the common denominator of being 'cost effective' is ensuring the quality of the data while keeping the number of measurements as small as possible.

The rapidly growing area of Compressed Sensing studies 'economical' data acquisition processes. We refer the reader to [9, 17] and to the book 23] for more information on the origins of Compressed Sensing and a survey of the progress that has been made in the area in recent years.

\footnotetext{
${ }^{1}$ CNRS, CMAP, Ecole Polytechnique, 91120 Palaiseau, France.

${ }^{2}$ Department of Mathematics, Technion, I.I.T, Haifa 32000, Israel.

${ }^{3}$ Email: guillaume.lecue@cmap.polytechnique.fr

${ }^{4}$ Email: shahar@tx.technion.ac.il

${ }^{5}$ Supported by the Mathematical Sciences Institute - The Australian National University and by ISF grant 900/10.
} 
At the heart of Compressed Sensing is a simple idea that has been a recurring theme in Mathematics and Statistics: while complex objects (in this case, data), live in high-dimensional spaces, they can be described effectively using low-dimensional, approximating structures; moreover, randomness may be used to expose these low-dimensional structures. Of course, unlike more theoretical applications of this idea, identifying the low-dimensional structures in the context of Compressed Sensing must be robust and efficient, otherwise, such procedures will be of little practical use.

In the standard Compressed Sensing setup, one observes linear measurements $y_{i}=\left\langle X_{i}, x_{0}\right\rangle, i=1, \ldots, N$ of an unknown vector $x_{0} \in \mathbb{R}^{n}$. To make the data acquisition process 'cost-effective', the number of measurements $N$ is assumed to be much smaller than the dimension $n$, and the goal is to identify $x_{0}$ using those measurements.

Because the resulting system of equations is under-determined, there is no hope, in general, of identifying $x_{0}$. However, if $x_{0}$ is believed to be well approximated by a low-dimensional structure, for example, if $x_{0}$ is supported on at most $s$ coordinates for some $s \leq N$, the problem becomes more feasible.

Let $\left(f_{1}, \ldots, f_{N}\right)$ be the canonical basis of $\mathbb{R}^{N}$ (we will later use $\left(e_{1}, \ldots, e_{n}\right)$ to denote the canonical basis of $\mathbb{R}^{n}$ ) and consider the matrix

$$
\Gamma=\frac{1}{\sqrt{N}} \sum_{i=1}^{N}\left\langle X_{i}, \cdot\right\rangle f_{i},
$$

called the measurement matrix. One possible recovery procedure is $\ell_{0^{-}}$ minimization, in which one selects a vector $t \in \mathbb{R}^{n}$ that has the shortest support among all vectors satisfying $\Gamma t=\Gamma x_{0}$. Unfortunately, $\ell_{0}$ minimization is known to be NP-hard in general (see [33] or Theorem 2.17 in [23]). Thus, even without analyzing if and when $\ell_{0}$-minimization actually recovers $x_{0}$, it is obvious that a more computationally reasonable procedure has to be found.

Fortunately, efficient procedures have been used since the seventies in geophysics (see, for instance [14, 42, 40], and Logan's Ph.D. thesis [28]). Those procedures are based on $\ell_{1}$-minimization for which early theoretical works can be found in [20] and [13].

In particular, Basis Pursuit is a convex relaxation of $\ell_{0}$-minimization, and since it can be recast as a linear program (see, e.g., Chapter 15 in [23]), it is far more reasonable than $\ell_{0}$-minimization from the computational viewpoint. 
Definition 1.1 Given the measurement matrix $\Gamma$ and the measurements $\Gamma x_{0}=\left(\left\langle X_{i}, x_{0}\right\rangle\right)_{i=1}^{N}$, Basis Pursuit returns a vector $\hat{x}$ that satisfies

$$
\hat{x} \in \operatorname{argmin}\left(\|t\|_{1}: \Gamma t=\Gamma x_{0}\right) .
$$

Since one may solve this minimization problem effectively, the focus may be shifted to the quality of the solution: whether one can identify measurement vectors $X_{1}, \ldots, X_{N}$ for which (1.1) has a unique solution, which is $x_{0}$ itself, for any $x_{0}$ that is $s$-sparse (i.e. supported on at most $s$ coordinates).

Definition 1.2 Let $\Sigma_{s}$ be the set of all s-sparse vectors in $\mathbb{R}^{n}$. An $N \times n$ matrix $\Gamma$ satisfies the exact reconstruction property of order $s$ if for every $x_{0} \in \Sigma_{s}$,

$$
\operatorname{argmin}\left(\|t\|_{1}: \Gamma t=\Gamma x_{0}\right)=\left\{x_{0}\right\} .
$$

It follows from Proposition 2.2.18 in [12] that if $\Gamma$ satisfies $\operatorname{ER}(s)$ then necessarily the number of measurements (rows) is at least $N \geq c_{0} s \log (\mathrm{en} / \mathrm{s})$, where $c_{0}$ is a suitable absolute constant. On the other hand, there are constructions of (random) matrices $\Gamma$ that satisfy $\operatorname{ER}(s)$ with $N$ proportional to $s \log (e n / s)$. From here on and with a minor abuse of notation, we will refer to $s \log (e n / s)$ as the optimal number of measurements and ignore the exact dependence on the constant $c_{0}$.

Unfortunately, the only matrices that are known to satisfy the reconstruction property with an optimal number of measurements are random - which is not surprising, as randomness is one of the most effective tools in exposing low-dimensional, approximating structures. A typical example of an 'optimal matrix' is the Gaussian matrix, which has independent standard normal random variables as entries. Other examples of optimal measurement matrices are $\Gamma=N^{-1 / 2} \sum_{i=1}^{N}\left\langle X_{i}, \cdot\right\rangle f_{i}$ where $X_{1}, \ldots, X_{N}$ are independent, isotropic and $L$-subgaussian random vectors:

Definition 1.3 A symmetric random vector $X \in \mathbb{R}^{n}$ is isotropic if for every $t \in \mathbb{R}^{n}, \mathbb{E}\langle X, t\rangle^{2}=\|t\|_{2}^{2}$; it is L-subgaussian if for every $t \in \mathbb{R}^{n}$ and every $p \geq 2,\|\langle X, t\rangle\|_{L_{p}} \leq L \sqrt{p}\|\langle X, t\rangle\|_{L_{2}}$.

The optimal behaviour of isotropic, $L$-subgaussian matrix ensembles and other ensembles like it, occurs because a typical matrix acts on $\Sigma_{s}$ in an isomorphic way when $N \geq c_{1} s \log (e n / s)$, and in the $L$-subgaussian case, $c_{1}$ is a constant that depends only on $L$. In Compressed Sensing literature, this isomorphic behaviour is called the Restricted Isometry property (RIP) (see, for example [8, 10, 32]): A matrix $\Gamma$ satisfies the RIP in $\Sigma_{s}$ with constant $0<\delta<1$, if for every $t \in \Sigma_{s}$,

$$
(1-\delta)\|t\|_{2} \leq\|\Gamma t\|_{2} \leq(1+\delta)\|t\|_{2} .
$$


It is straightforward to show that if $\Gamma$ satisfies the RIP in $\Sigma_{2 s}$ for a sufficiently small constant $\delta$, then it has the exact reconstruction property of order $s$ (see, e.g. [9, 10, 7]).

The standard proof of the RIP for subgaussian ensembles is based on the rapid tail decay of linear functionals $\langle X, t\rangle$. Thus, it seemed natural to ask whether the RIP holds even when linear functionals exhibit a slower decay - for example, when $X$ is $L$-subexponential - that is, when linear functionals only satisfy that $\|\langle X, t\rangle\|_{L_{p}} \leq L p\|\langle X, t\rangle\|_{L_{2}}$ for every $t \in \mathbb{R}^{n}$ and every $p \geq 2$.

Proving the RIP for subexponential ensembles is a much harder task than for subgaussian ensembles (see, e.g. [1]). Moreover, the RIP does not exhibit the same optimal quantitative behaviour as in the Gaussian case: it holds with high probability only when $N \geq c_{2}(L) s \log ^{2}(e n / s)$, and this estimate cannot be improved, as can be seen when $X$ has independent, symmetric exponential random variables as coordinates [1].

Although the RIP need not be true for isotropic $L$-subexponential ensemble using the optimal number of measurements, results in [24] (see Theorem 7.3 there) and in 22 show that exact reconstruction can still be achieved by such an ensemble and with the optimal number of measurements. This opens the door to an intriguing question: whether considerably weaker assumptions on the measurement vector may still lead to Exact Reconstruction even when the RIP fails.

The main result presented here does just that, using the small-ball method introduced in [30, 31].

Definition 1.4 A random vector $X$ satisfies the small-ball condition in the set $\Sigma_{s}$ with constants $u, \beta>0$ if for every $t \in \Sigma_{s}$,

$$
P\left(|\langle X, t\rangle|>u\|t\|_{2}\right) \geq \beta .
$$

The small-ball condition is a rather minimal assumption on the measurement vector and is satisfied in fairly general situations for values of $u$ and $\beta$ that are suitable constants, independent of the dimension $n$.

Under some normalization (like isotropicity), a small-ball condition is an immediate outcome of the Paley-Zygmund inequality (see, e.g. [15]) and moment equivalence. For example, in the following cases a small-ball condition holds with constants that depend only on $\kappa_{0}$ (and on $\varepsilon$ for the first case); the straightforward proof may be found in [30].

- $X$ is isotropic and for every $t \in \Sigma_{s},\|\langle X, t\rangle\|_{L_{2+\varepsilon}} \leq \kappa_{0}\|\langle X, t\rangle\|_{L_{2}}$ for some $\varepsilon>0$; 
- $X$ is isotropic and for every $t \in \Sigma_{s},\|\langle X, t\rangle\|_{L_{2}} \leq \kappa_{0}\|\langle X, t\rangle\|_{L_{1}}$.

Because the small-ball condition means that marginals of $X$ do not assign too much weight close to 0 , it may hold even without integrability (and in particular, $X$ need not have a covariance matrix). One such example is a random vector whose coordinates are independent random variables that are absolutely continuous with respect to the Lebesgue measure and with a density almost surely bounded by $\kappa_{0}$. Indeed, as noted in Theorem 1.2 from [38], for every $t \in \mathbb{R}^{n},\left\langle X, t /\|t\|_{2}\right\rangle$ has a density that is almost surely bounded by $\sqrt{2} \kappa_{0}$. In particular, $P\left(|\langle X, t\rangle| \geq\left(4 \sqrt{2} \kappa_{0}\right)^{-1}\|t\|_{2}\right) \geq 1 / 2$ and $X$ satisfies the small ball condition with $u=\left(4 \sqrt{2} \kappa_{0}\right)^{-1}$ and $\beta=1 / 2$. The estimate on the density of $\left\langle X, t /\|t\|_{2}\right\rangle$ follows by combining a result due to B. Rogozin [36] on the maximal value of a convolution product of densities, and a result due to $\mathrm{K}$. Ball [2], on the maximal volume of a section of the cube $[-1 / 2,1 / 2]^{n}$.

Our first result shows that a combination of the small-ball condition and a weak moment assumption suffices to ensure the exact reconstruction property with the optimal number of measurements.

Theorem A. There exist absolute constants $c_{0}, c_{1}$ and $c_{2}$ and for every $\alpha \geq 1 / 2$ there exists a constant $c_{3}(\alpha)$ that depends only on $\alpha$ for which the following holds. Let $X=\left(x_{i}\right)_{i=1}^{n}$ be a random vector on $\mathbb{R}^{n}$ (with potentially dependent coordinates). Assume that

1. there are $\kappa_{1}, \kappa_{2}, w>1$ that satisfy that for every $1 \leq j \leq n,\left\|x_{j}\right\|_{L_{2}}=1$ and, for every $4 \leq p \leq 2 \kappa_{2} \log (w n),\left\|x_{j}\right\|_{L_{p}} \leq \kappa_{1} p^{\alpha}$.

2. $X$ satisfies the small ball condition in $\Sigma_{s}$ with constants $u$ and $\beta$.

If

$$
N \geq c_{0} \max \left\{s \log \left(\frac{e n}{s}\right),\left(c_{3}(\alpha) \kappa_{1}^{2}\right)^{2}\left(\kappa_{2} \log (w n)\right)^{\max \{4 \alpha-1,1\}}\right\},
$$

and $X_{1}, \ldots, X_{N}$ are independent copies of $X$, then, with probability at least

$$
1-2 \exp \left(-c_{1} \beta^{2} N\right)-1 / w^{\kappa_{2}} n^{\kappa_{2}-1},
$$

$\Gamma=N^{-1 / 2} \sum_{i=1}^{N}\left\langle X_{i}, \cdot\right\rangle f_{i}$ satisfies the exact reconstruction property in $\Sigma_{s_{1}}$ for $s_{1}=c_{2} u^{2} \beta s$.

An immediate outcome of Theorem A is the following: 
- Let $x$ be a centered random variable that has variance 1 and for which $\|x\|_{L_{p}} \leq c \sqrt{p}$ for $1 \leq p \leq 2 \log n$. If $X$ has independent coordinates distributed as $x$, then the corresponding matrix $\Gamma$ with $N \geq$ $c_{1} s \log (e n / s)$ rows can be used as a measurement matrix and recover any $s$-sparse vector with large probability.

It is relatively straightforward to derive many other results of a similar flavour, leading to random ensembles that satisfy the exact reconstruction property with the optimal number of measurements.

Remark 1.5 Our focus is on measurement matrices with independent rows, that satisfy conditions of a stochastic nature - they have i.i.d. rows. Other types of measurement matrices that have some structure have also been used in Compressed Sensing. One notable example is a random Fourier measurement matrix, obtained by randomly selecting rows from the discrete Fourier matrix (see, e.g. [10], [37] or Chapter 12 in [23]).

One may wonder if the small-ball condition is satisfied for more structured matrices, as the argument we use here does not extend immediately to such cases. And, indeed, for structured ensembles one may encounter a different situation: a small-ball condition that is not uniform, in the sense that the constants $u$ and $\beta$ from Definition 1.4 are direction-dependent. Moreover, in some cases, the known estimates on these constants are far from what is expected.

Results of the same flavour of Theorem A may follow from a 'good enough' small-ball condition, even if it is not uniform, by slightly modifying the argument we use here. However, obtaining a satisfactory 'non-uniform' small-ball condition is a different story. For example, in the Fourier case, such an estimate is likely to require quantitative extensions of the LittlewoodPaley theory - a worthy challenge in its own right, and one which goes far beyond the goals of this article.

Just as noted for subexponential ensembles, Theorem A cannot be proved using an RIP-based argument. A key ingredient in the proof is the following observation:

Theorem B. Let $\Gamma: \mathbb{R}^{n} \mapsto \mathbb{R}^{N}$ and denote by $\left(e_{1}, \ldots, e_{n}\right)$ the canonical basis of $\mathbb{R}^{n}$. Assume that:

a) for every $x \in \Sigma_{s},\|\Gamma x\|_{2} \geq c_{0}\|x\|_{2}$, and

b) for every $j \in\{1, \ldots, n\},\left\|\Gamma e_{j}\right\|_{2} \leq c_{1}$. 
Setting $s_{1}=\left\lfloor\left(c_{0}^{2}(s-1)\right) /\left(4 c_{1}^{2}\right)\right\rfloor-1, \Gamma$ satisfies the exact reconstruction property in $\Sigma_{s_{1}}$.

Compared with the RIP, conditions a) and b) in Theorem B are weaker, as it suffices to verify the right-hand side of (1.2) for 1-sparse vectors rather than for every $s$-sparse vector. This happens to be a substantial difference: the assumption that for every $t \in \Sigma_{s},\|\Gamma t\|_{2} \leq(1+\delta)\|t\|_{2}$ is a costly one, and happens to be the reason for the gap between the RIP and the exact reconstruction property. Indeed, while the lower bound in the RIP holds for rather general ensembles (see [30] and the next section for more details), and is guaranteed solely by the small-ball condition, the upper bound is almost equivalent to having the coordinates of $X$ exhibit a subgaussian behaviour of moments, at least up to some level. Even the fact that one has to verify the upper bound for 1-sparse vectors comes at a cost, namely, the moment assumption (1) in Theorem A.

The second goal of this note is to illustrate that while Exact Reconstruction is 'cheaper' than the RIP, it still comes at a cost - namely, that the moment condition (1) in Theorem A is truly needed.

Definition 1.6 $A$ random matrix $\Gamma$ is generated by the random variable $x$ if $\Gamma=N^{-1 / 2} \sum_{i=1}^{N}\left\langle X_{i}, \cdot\right\rangle f_{i}$ and $X_{1}, \ldots, X_{N}$ are independent copies of the random vector $X=\left(x_{1}, \ldots, x_{n}\right)^{\top}$ whose coordinates are independent copies of $x$.

Theorem C. There exist absolute constants $c_{0}, c_{1}, c_{2}$ and $c_{3}$ for which the following holds. Given $n \geq c_{0}$ and $N \log N \leq c_{1} n$, there exists a mean-zero, variance one random variable $x$ with the following properties:

- $\|x\|_{L_{p}} \leq c_{2} \sqrt{p}$ for $2<p \leq c_{3}(\log n) /(\log N)$.

- If $\left(x_{j}\right)_{j=1}^{n}$ are independent copies of $x$ then $X=\left(x_{1}, \ldots, x_{n}\right)^{\top}$ satisfies the small-ball condition with constants $u$ and $\beta$ that depend only on $c_{2}$.

- Denote by $\Gamma$ the $N \times n$ matrix generated by $x$. For every $k \in\{1, \ldots, n\}$, with probability larger than $1 / 2$, argmin $\left(\|t\|_{1}: \Gamma t=\Gamma e_{k}\right) \neq\left\{e_{k}\right\}$; therefore, $e_{k}$ is not exactly reconstructed by Basis Pursuit and so $\Gamma$ does not satisfy the exact reconstruction property of order 1.

To put Theorem $\mathrm{C}$ in some perspective, note that if $\Gamma$ is generated by $x$ for which $\|x\|_{L_{2}}=1$ and $\|x\|_{L_{p}} \leq c_{4} \sqrt{p}$ for $2<p \leq c_{5} \log n$, then $X=$ 
$\left(x_{i}\right)_{i=1}^{n}$ satisfies the small-ball condition with constants that depend only on $c_{4}$, and by Theorem A, if $N \geq c_{6} \log n, \Gamma$ satisfies $\operatorname{ER}(1)$ with high probability. On the other hand, the random ensemble from Theorem $\mathrm{C}$ is generated by $x$ that has almost identical properties - with one exception: its $L_{p}$ norm is well behaved only for $p \leq c_{7}(\log n) / \log \log n$. This small gap in the number of moments has a significant impact: with probability at least $1 / 2, \Gamma$ does not satisfy $\operatorname{ER}(1)$ when $N$ is of the order of $\log n$.

Therefore, the moment condition in Theorem A is indeed required (up to a $\log \log n$ factor).

The idea behind the proof of Theorem $\mathrm{C}$ is to construct a random matrix $\Gamma$ for which, given any basis vector $e_{k}$, with probability at least $1 / 2,\left\|\Gamma e_{k}\right\|_{2} \leq$ 1 , while the set $\left\{\Gamma e_{j}, j \neq k\right\}$ has many 'very spiky' vectors: the convex hull $\operatorname{conv}\left( \pm \Gamma e_{j}, j \neq k\right)$ contains a perturbation of $2 \sqrt{N} B_{1}^{N}$, i.e., a large multiple of the unit ball in $\ell_{1}^{N}$. Since such a set must contain the Euclidean unit ball, and in particular, $\Gamma e_{k}$ as well, it follows that $e_{k}$ cannot be the unique solution of the $\ell_{1}$ minimization problem $\min \left(\|t\|_{1}: \Gamma t=\Gamma e_{k}\right)$.

The fact that the coordinates of $X$ do not have enough well behaved moments is the key feature that allows one to generate many 'spiky' columns in a typical $\Gamma$.

An alternative formulation of Theorem $\mathrm{C}$ is the following:

Theorem $\mathbf{C}^{\prime}$. There are absolute constants $c_{0}, c_{1}, c_{2}$ and $\kappa$ for which the following holds. If $n \geq c_{0}$ and $2<p<c_{1} \log n$, there exists a mean-zero and variance 1 random variable $x$, for which $\|x\|_{L_{q}} \leq \kappa \sqrt{q}$ for $2<q \leq p$, and if $N \leq c_{2} \sqrt{p}(n / \log n)^{1 / p}$ and $\Gamma$ is the $N \times n$ matrix generated by $x$, then with probability at least $1 / 2, \Gamma$ does not satisfy the exact reconstruction property of order 1 .

Theorem $\mathrm{C}$ and Theorem $\mathrm{C}^{\prime}$ imply that Basis Pursuit may perform poorly when the coordinates of $X$ do not have enough moments, and requires a polynomial number of measurements in $n$ to ensure Exact Reconstruction. This happens to be the price of convex relaxation: a rather striking observation is that $\ell_{0}$-minimization achieves recovery with the optimal number of measurements under an even weaker small-ball condition than in Theorem A, and without any additional moment assumptions.

Recall that $\ell_{0}$-minimization is defined by $\hat{x}=\operatorname{argmin}\left(\|t\|_{0}: \Gamma t=\Gamma x_{0}\right)$, where $\|t\|_{0}$ is the cardinality of the support of $t$.

Definition 1.7 X satisfies a weak small-ball condition in $\Sigma_{s}$ with constant 
$\beta$ if for every $t \in \Sigma_{s}$,

$$
P(|\langle X, t\rangle|>0) \geq \beta
$$

Theorem D. For every $0<\beta<1$ there exist constants $c_{0}$ and $c_{1}$ that depend only on $\beta$ and for which the following holds. Let $X$ be a random vector that satisfies the weak small-ball condition in $\Sigma_{s}$ with a constant $\beta$. Let $X_{1}, \ldots, X_{N}$ be $N$ independent copies of $X$ and set $\Gamma=N^{-1 / 2} \sum_{i=1}^{N}\left\langle X_{i}, \cdot\right\rangle f_{i}$. If $N \geq c_{0} s \log (e n / s)$ then with probability at least $1-2 \exp \left(-c_{1} N\right)$, for every $x_{0} \in \Sigma_{\lfloor s / 2\rfloor}, \ell_{0}$-minimization has a unique solution, which is $x_{0}$ itself.

The price of convex relaxation can now be clearly seen through the number of measurements needed for exact reconstruction: consider the random vector $X$ constructed in Theorem $\mathrm{C}^{\prime}$ for, say, $p=4$. Since $X$ satisfies the conditions of Theorem $\mathrm{D}, \ell_{0}$ minimization may be used to recover any $s$-sparse vector with only $N=c s \log (\mathrm{en} / \mathrm{s})$ random measurements. In contrast, Basis Pursuit requires at least $\sim(n / \log n)^{1 / 4}$ measurements to reconstruct 1-sparse vectors.

It should be noted that under much stronger assumptions on $X$, the exact recovery of $s$-sparse vectors using $\ell_{0}$-minimization may occur when $N$ is as small as $2 s$. Indeed, it suffices to ensure that all the $N \times 2 s$ sub-matrices of $\Gamma$ are non-singular, and this is the case when $N=2 s$ if the entries of $\Gamma$ are independent random variables that are absolutely continuous (see Chapter 2 in [23] for more details).

We end this introduction with a word about notation and the organization of the article. The proofs of Theorem A, Theorem B and Theorem D are presented in the next section, while the proofs of Theorem $\mathrm{C}$ and Theorem $\mathrm{C}^{\prime}$ may be found in Section 3 . The final section is devoted to results in a natural 'noisy' extension of Compressed Sensing. In particular, we prove that both the Compatibility Condition and the Restricted Eigenvalue Condition hold under weak moment assumptions; we also study related properties of random polytopes.

As for notation, throughout, absolute constants or constants that depend on other parameters are denoted by $c, C, c_{1}, c_{2}$, etc., (and, of course, we will specify when a constant is absolute and when it depends on other parameters). The values of these constants may change from line to line. The notation $x \sim y$ (resp. $x \lesssim y$ ) means that there exist absolute constants $0<c<C$ for which $c y \leq x \leq C y$ (resp. $x \leq C y$ ). If $b>0$ is a parameter then $x \lesssim_{b} y$ means that $x \leq C(b) y$ for some constant $C(b)$ that depends only on $b$. 
Let $\ell_{p}^{m}$ be $\mathbb{R}^{m}$ endowed with the norm $\|x\|_{\ell_{p}^{m}}=\left(\sum_{j}\left|x_{j}\right|^{p}\right)^{1 / p}$; the corresponding unit ball is denoted by $B_{p}^{m}$ and the unit Euclidean sphere in $\mathbb{R}^{m}$ is $S^{m-1}$. If $A \subset \mathbb{R}^{n}$ then $\mathbb{1}_{A}$ denotes the indicator function of $A$. Finally, we will assume that $(\mathcal{X}, \mu)$ is a probability space, and that $X$ is distributed according to $\mu$.

\section{Proof of Theorem A, B and D}

The proof of Theorem A has several components, and although the first of which is rather standard, we present it for the sake of completeness.

Lemma 2.1 Let $\Gamma: \mathbb{R}^{n} \rightarrow \mathbb{R}^{N}$ be a matrix and set $\operatorname{ker}(\Gamma)$ to be its kernel. If $0<r<1$ and $B_{1}^{n} \cap r S^{n-1}$ does not intersect $\operatorname{ker}(\Gamma)$, then $\Gamma$ satisfies the exact reconstruction property in $\Sigma_{\left\lfloor(2 r)^{-2}\right\rfloor}$.

Proof. Observe that if $x \in B_{1}^{n}$ and $\|x\|_{2} \geq r$ then $y=r x /\|x\|_{2} \in B_{1}^{n} \cap$ $r S^{n-1}$. Therefore, if $y \notin \operatorname{ker}(\Gamma)$, the same holds for $x$; thus

$$
\sup _{x \in B_{1}^{n} \cap \operatorname{ker}(\Gamma)}\|x\|_{2}<r .
$$

Let $s=\left\lfloor(2 r)^{-2}\right\rfloor$, fix $x_{0} \in \Sigma_{s}$ and put $I$ to be the set indices of coordinates on which $x_{0}$ is supported. Given a nonzero $h \in \operatorname{ker}(\Gamma)$, let $h=h_{I}+h_{I^{c}}$ - the decomposition of $h$ to coordinates in $I$ and in $I^{c}$. Since $h /\|h\|_{1} \in B_{1}^{n} \cap \operatorname{ker}(\Gamma)$, it follows that $\|h\|_{2}<r\|h\|_{1}$, and by the choice of $s, 2 \sqrt{s}\|h\|_{2}<\|h\|_{1}$. Therefore,

$$
\begin{aligned}
\left\|x_{0}+h\right\|_{1} & =\left\|x_{0}+h_{I}\right\|_{1}+\left\|h_{I^{c}}\right\|_{1} \geq\left\|x_{0}\right\|_{1}-\left\|h_{I}\right\|_{1}+\left\|h_{I^{c}}\right\|_{1} \\
& =\left\|x_{0}\right\|_{1}-2\left\|h_{I}\right\|_{1}+\|h\|_{1} \geq\left\|x_{0}\right\|_{1}-2 \sqrt{|I|}\left\|h_{I}\right\|_{2}+\|h\|_{1}>\left\|x_{0}\right\|_{1} .
\end{aligned}
$$

Hence, $\left\|x_{0}+h\right\|_{1}>\left\|x_{0}\right\|_{1}$ and $x_{0}$ is the unique minimizer of the basis pursuit algorithm.

The main ingredient in the proof of Theorem A is Lemma 2.3 below, which is based on the small-ball method introduced in [30, 31. To formulate the lemma, one requires the notion of a VC class of sets.

Definition 2.2 Let $\mathcal{G}$ be a class of $\{0,1\}$-valued functions defined on a set $\mathcal{X}$. The set $\mathcal{G}$ is a $V C$-class if there exists an integer $V$ for which, given any $x_{1}, \ldots, x_{V+1} \in \mathcal{X}$

$$
\left|\left\{\left(g\left(x_{1}\right), \ldots, g\left(x_{V+1}\right)\right): g \in \mathcal{G}\right\}\right|<2^{V+1} .
$$

The $V C$-dimension of $\mathcal{G}$, denoted by $V C(\mathcal{G})$, is the smallest integer $V$ for which (2.1) holds. 
The VC dimension is a combinatorial complexity measure that may be used to control the $L_{2}(\mu)$-covering numbers of the class; indeed, set $N\left(\mathcal{G}, \varepsilon, L_{2}(\mu)\right)$ to be the smallest number of open balls of radius $\varepsilon$ relative to the $L_{2}(\mu)$ norm that are needed to cover $\mathcal{G}$. A well known result due to Dudley [21] is that if $V C(\mathcal{G})=V$ and $\mu$ is a probability measure on $\mathcal{X}$ then for every $0<\varepsilon<1$,

$$
N\left(\mathcal{G}, \varepsilon, L_{2}(\mu)\right) \leq\left(\frac{c_{1}}{\varepsilon}\right)^{c_{2} V},
$$

where $c_{1}$ and $c_{2}$ are absolute constants.

Lemma 2.3 There exist absolute constants $c_{1}$ and $c_{2}$ for which the following holds. Let $\mathcal{F}$ be a class of functions and assume that there are $\beta>0$ and $u \geq 0$ for which

$$
\inf _{f \in \mathcal{F}} P(|f(X)|>u) \geq \beta .
$$

Let $\mathcal{G}_{u}=\left\{\mathbb{1}_{\{|f|>u\}}: f \in \mathcal{F}\right\}$. If $V C\left(\mathcal{G}_{u}\right) \leq d$ and $N \geq c_{1} d / \beta^{2}$ then with probability at least $1-\exp \left(-c_{2} \beta^{2} N\right)$,

$$
\inf _{f \in \mathcal{F}}\left|\left\{i \in\{1, \ldots, N\}:\left|f\left(X_{i}\right)\right|>u\right\}\right| \geq \frac{\beta N}{2} .
$$

Remark 2.4 Note that $u=0$ is a 'legal choice' in Lemma 2.3, a fact that will be used in the proof of Theorem $D$.

Proof. Let $G\left(X_{1}, \ldots, X_{N}\right)=\sup _{g \in \mathcal{G}_{u}}\left|N^{-1} \sum_{i=1}^{N} g\left(X_{i}\right)-\mathbb{E} g(X)\right|$. By the bounded differences inequality (see, for example, Theorem 6.2 in [5]), with probability at least $1-\exp (-t)$,

$$
G\left(X_{1}, \ldots, X_{N}\right) \leq \mathbb{E} G\left(X_{1}, \ldots, X_{N}\right)+c_{1} \sqrt{\frac{t}{N}} .
$$

Standard empirical processes arguments (symmetrization, the fact that Bernoulli processes are subgaussian and the entropy estimate (2.2) - see, for example, Chapters 2.2, 2.3 and 2.6 in [47]), show that since $V C(\mathcal{G}) \leq d$,

$$
\mathbb{E} G\left(X_{1}, \ldots, X_{N}\right) \leq c_{2} \sqrt{\frac{d}{N}} \leq \frac{\beta}{4},
$$

provided that $N \gtrsim d / \beta^{2}$. Therefore, taking $t=N \beta^{2} / 16 c_{1}^{2}$, it follows that with probability at least $1-\exp \left(-c_{3} \beta^{2} N\right)$, for every $f \in \mathcal{F}$,

$$
\frac{1}{N} \sum_{i=1}^{N} \mathbb{1}_{\{|f|>u\}}\left(X_{i}\right) \geq P(|f(X)|>u)-\frac{\beta}{2} \geq \frac{\beta}{2} .
$$

Therefore, on that event, $\left|\left\{i:\left|f\left(X_{i}\right)\right|>u\right\}\right| \geq \beta N / 2$ for every $f \in \mathcal{F}$. 
Corollary 2.5 There exist absolute constants $c_{1}$ and $c_{2}$ for which the following holds. Let $X \in \mathbb{R}^{n}$ be a random vector.

1. If there are $0<\beta \leq 1$ and $u \geq 0$ for which $P(|\langle t, X\rangle|>u) \geq \beta$ for every $t \in S^{n-1}$ and if $N \geq c_{1} n / \beta^{2}$, then with probability at least $1-\exp \left(-c_{2} N \beta^{2}\right)$

$$
\inf _{t \in S^{n-1}} \frac{1}{N} \sum_{i=1}^{N}\left\langle X_{i}, t\right\rangle^{2}>\frac{u^{2} \beta}{2} .
$$

2. If there are $0<\beta \leq 1$ and $u \geq 0$ for which $P(|\langle t, X\rangle|>u) \geq \beta$ for every $t \in \Sigma_{s} \cap S^{n-1}$ and if $N \geq c_{1} s \log (e n / s) / \beta^{2}$, then with probability at least $1-\exp \left(-c_{2} N \beta^{2}\right)$,

$$
\inf _{t \in \Sigma_{s} \cap S^{n-1}} \frac{1}{N} \sum_{i=1}^{N}\left\langle X_{i}, t\right\rangle^{2}>\frac{u^{2} \beta}{2} .
$$

Remark 2.6 Note that the first part of Corollary 2.5 gives an estimate on the smallest singular value of the random matrix $\Gamma=N^{-1 / 2} \sum_{i=1}^{N}\left\langle X_{i}, \cdot\right\rangle f_{i}$. The proof follows the same path as in [25], but unlike the latter, no assumption on the covariance structure of $X$, used both in [25] and in [41], is required. In fact, Corollary 2.5 may be applied even if the covariance matrix does not exist. Thus, under a small-ball condition, the smallest singular value of $\Gamma$ is larger than $c(\beta, u)$ with high (exponential) probability.

Proof of Corollary 2.5. To prove the first part of the claim, let $\mathcal{F}=$ $\left\{\langle t, \cdot\rangle: t \in S^{n-1}\right\}$. Recall that the VC dimension of a class of half-spaces in $\mathbb{R}^{n}$ is at most $n$, and thus, one may verify that for every $u \geq 0$, the VC dimension of

$$
\mathcal{G}_{u}=\left\{\mathbb{1}_{\{|f|>u\}}: f \in \mathcal{F}\right\}
$$

is at most $c_{1} n$ for a suitable absolute constant $c_{1}$ (see, e.g., Chapter 2.6 in 47]). The claim now follows immediately from Lemma 2.3 because

$$
\frac{1}{N} \sum_{i=1}^{N}\left\langle t, X_{i}\right\rangle^{2}>\frac{u^{2}}{N}\left|\left\{i:\left|\left\langle X_{i}, t\right\rangle\right|>u\right\}\right|
$$

for every $t \in S^{n-1}$. 
Turning to the second part, note that $\Sigma_{s} \cap S^{n-1}$ is a union of $\left(\begin{array}{l}n \\ s\end{array}\right)$ spheres of dimension $s$. Applying the first part to each one of those spheres, combined with the union bound, it follows that for $N \geq c_{2} \beta^{-2} s \log (e n / s)$, with probability at least $1-\exp \left(-c_{3} N \beta^{2}\right)$,

$$
\inf _{t \in \Sigma_{s} \cap S^{n-1}} \frac{1}{N} \sum_{i=1}^{N}\left\langle X_{i}, t\right\rangle^{2}>\frac{u^{2} \beta}{2} .
$$

Corollary 2.5 shows that the small-ball condition for linear functionals implies that $\Gamma$ 'acts well' on $s$-sparse vectors. However, according to Lemma 2.1. exact recovery is possible if $\Gamma$ is well behaved on the set

$$
\sqrt{\kappa_{0} s} B_{1}^{n} \cap S^{n-1}=\left\{x \in \mathbb{R}^{n}:\|x\|_{1} \leq \sqrt{\kappa_{0} s}, \quad\|x\|_{2}=1\right\}
$$

for a well-chosen constant $\kappa_{0}$. In the standard (RIP-based) argument, one proves exact reconstruction by first showing that the RIP holds in $\Sigma_{s}$, and then the fact that each vector in $\sqrt{\kappa_{0} s} B_{1}^{n} \cap S^{n-1}$ is well approximated by vectors from $\Sigma_{s}$ (see, for instance, [12]) allows one to extend the RIP from $\Sigma_{s}$ to $\sqrt{\kappa_{0} s} B_{1}^{n} \cap S^{n-1}$. Unfortunately, this extension requires both upper and lower estimates in the RIP.

Since the upper part of the RIP in $\Sigma_{s}$ forces severe restrictions on the random vector $X$, one has to resort to a totally different argument if one wishes to extend the lower bound from $\Sigma_{s}$ (which only requires the small-ball condition) to $\sqrt{\kappa_{0} s} B_{1}^{n} \cap S^{n-1}$.

The method presented below is based on Maurey's empirical method and has been recently used in 34.

Lemma 2.7 Let $\Gamma: \mathbb{R}^{n} \rightarrow \mathbb{R}^{N}$, put $1<s \leq n$ and assume that for every $x \in \Sigma_{s},\|\Gamma x\|_{2} \geq \lambda\|x\|_{2}$. If $y \in \mathbb{R}^{n}$ is a nonzero vector and $\mu_{j}=\left|y_{j}\right| /\|y\|_{1}$, then

$$
\|\Gamma y\|_{2}^{2} \geq \lambda^{2}\|y\|_{2}^{2}-\frac{\|y\|_{1}^{2}}{s-1}\left(\sum_{j=1}^{n}\left\|\Gamma e_{j}\right\|_{2}^{2} \mu_{j}-\lambda^{2}\right) .
$$

Proof. Fix $y \in \mathbb{R}^{n}$, let $Y$ be a random vector in $\mathbb{R}^{n}$ defined by

$$
P\left(Y=\|y\|_{1} \operatorname{sgn}\left(y_{j}\right) e_{j}\right)=\left|y_{j}\right| /\|y\|_{1},
$$

for every $j=1, \ldots, n$ and observe that $\mathbb{E} Y=y$. 
Let $Y_{1}, \ldots, Y_{s}$ be independent copies of $Y$ and set $Z=s^{-1} \sum_{k=1}^{s} Y_{k}$. Note that $Z \in \Sigma_{s}$ for every realization of $Y_{1}, \ldots, Y_{s}$; thus $\|\Gamma Z\|_{2}^{2} \geq \lambda^{2}\|Z\|_{2}^{2}$ and

$$
\mathbb{E}\|\Gamma Z\|_{2}^{2} \geq \lambda^{2} \mathbb{E}\|Z\|_{2}^{2} .
$$

It is straightforward to verify that $\mathbb{E}\langle Y, Y\rangle=\|y\|_{1}^{2}$; that if $i \neq j$ then $\mathbb{E}\left\langle\Gamma Y_{i}, \Gamma Y_{j}\right\rangle=\langle\Gamma y, \Gamma y\rangle$; and that for every $1 \leq k \leq s$,

$$
\mathbb{E}\left\langle\Gamma Y_{k}, \Gamma Y_{k}\right\rangle=\|y\|_{1} \sum_{j=1}^{n}\left|y_{j}\right|\left\|\Gamma e_{j}\right\|_{2}^{2}
$$

Therefore, setting $\mu_{j}=\left|y_{j}\right| /\|y\|_{1}$ and $W=\sum_{j=1}^{n}\left\|\Gamma e_{j}\right\|_{2}^{2} \mu_{j}$,

$$
\begin{aligned}
\mathbb{E}\|\Gamma Z\|_{2}^{2} & =\frac{1}{s^{2}} \sum_{i, j=1}^{s} \mathbb{E}\left\langle\Gamma Y_{i}, \Gamma Y_{j}\right\rangle=\left(1-\frac{1}{s}\right)\|\Gamma y\|_{2}^{2}+\frac{\|y\|_{1}}{s} \sum_{j=1}^{n}\left|y_{j}\right|\left\|\Gamma e_{j}\right\|_{2}^{2} \\
& =\left(1-\frac{1}{s}\right)\|\Gamma y\|_{2}^{2}+W \frac{\|y\|_{1}^{2}}{s}
\end{aligned}
$$

and using the same argument one may show that

$$
\mathbb{E}\|Z\|_{2}^{2}=\left(1-\frac{1}{s}\right)\|y\|_{2}^{2}+\frac{\|y\|_{1}^{2}}{s} .
$$

Combining these two estimates with (2.4),

$$
\left(1-\frac{1}{s}\right)\|\Gamma y\|_{2}^{2} \geq \lambda^{2}\left(\left(1-\frac{1}{s}\right)\|y\|_{2}^{2}+\frac{\|y\|_{1}^{2}}{s}\right)-W \frac{\|y\|_{1}^{2}}{s},
$$

proving the claim.

Proof of Theorem B: Assume that for every $x \in \Sigma_{s},\|\Gamma x\|_{2} \geq c_{0}\|x\|_{2}$ and that for every $1 \leq i \leq n,\left\|\Gamma e_{i}\right\|_{2} \leq c_{1}$. It follows from Lemma 2.7 that if $s-1>c_{1}^{2} /\left(c_{0}^{2} r^{2}\right)$, then for every $y \in B_{1}^{n} \cap r S^{n-1}$,

$$
\|\Gamma y\|_{2}^{2} \geq c_{0}^{2}\|y\|_{2}^{2}-\frac{\|y\|_{1}}{s-1} \sum_{i=1}^{n}\left\|\Gamma e_{i}\right\|_{2}^{2}\left|y_{i}\right| \geq c_{0}^{2} r^{2}-\frac{c_{1}^{2}}{s-1}>0 .
$$

The claim now follows from Lemma 2.1.

Consider the matrix $\Gamma=N^{-1 / 2} \sum_{i=1}^{N}\left\langle X_{i}, \cdot\right\rangle f_{i}$. Observe that for every $t \in \mathbb{R}^{n},\|\Gamma t\|_{2}^{2}=N^{-1} \sum_{i=1}^{N}\left\langle X_{i}, t\right\rangle^{2}$, and that if $X_{j}=\left(x_{i, j}\right)_{i=1}^{n}$ then

$$
\left\|\Gamma e_{j}\right\|_{2}^{2}=\frac{1}{N} \sum_{i=1}^{N} x_{i, j}^{2}
$$


which is an average of $N$ iid random variables (though $\left\|\Gamma e_{1}\right\|_{2}, \ldots,\left\|\Gamma e_{n}\right\|_{2}$ need not be independent).

Thanks to Theorem B and Corollary 2.5, the final component needed for the proof of Theorem A is information on the sum of iid random variables, which will be used to bound $\max _{1 \leq j \leq n}\left\|\Gamma e_{j}\right\|_{2}^{2}$ from above.

Lemma 2.8 There exists an absolute constant $c_{0}$ for which the following holds. Let $z$ be a mean-zero random variable and put $z_{1}, \ldots, z_{N}$ to be $N$ independent copies of $z$. Let $p_{0} \geq 2$ and assume that there exists $\kappa_{1}>0$ and $\alpha \geq 1 / 2$ for which $\|z\|_{L_{p}} \leq \kappa_{1} p^{\alpha}$ for every $2 \leq p \leq p_{0}$. If $N \geq p_{0}^{\max \{2 \alpha-1,1\}}$ then for every $2 \leq p \leq p_{0}$,

$$
\left\|\frac{1}{\sqrt{N}} \sum_{i=1}^{N} z_{i}\right\|_{L_{p}} \leq c_{1}(\alpha) \kappa_{1} \sqrt{p}
$$

where $c_{1}(\alpha)=c_{0} \exp ((2 \alpha-1))$.

Lemma 2.8 shows that even under a weak moment assumption, namely that $\|z\|_{L_{p}} \lesssim p^{\alpha}$ for $p \leq p_{0}$ and $\alpha \geq 1 / 2$ that can be large, a normalized sum of $N$ independent copies of $z$ exhibits a 'subgaussian' moment growth up to the same $p_{0}$, as long as $N$ is sufficiently large.

The proof of Proposition 2.8 is based on the following fact due to Latała.

Theorem 2.9 ([26], Theorem 2 and Remark 2) If $z$ is a mean-zero random variable and $z_{1}, \ldots, z_{N}$ are independent copies of $z$, then for any $p \geq 2$,

$$
\left\|\sum_{i=1}^{N} z_{i}\right\|_{L_{p}} \sim \sup \left\{\frac{p}{s}\left(\frac{N}{p}\right)^{1 / s}\|z\|_{L_{s}}: \max \{2, p / N\} \leq s \leq p\right\} .
$$

Proof of Lemma 2.8. Let $2 \leq p \leq p_{0}$ and $N \geq p$. Since $\|z\|_{L_{s}} \leq \kappa_{1} s^{\alpha}$ for any $2 \leq s \leq p$, it follows from Theorem 2.9 that

$$
\left\|\sum_{i=1}^{N} z_{i}\right\|_{L_{p}} \leq c_{0} \kappa_{1} \sup \left\{p(N / p)^{1 / s} s^{-1+\alpha}: \max \{2, p / N\} \leq s \leq p\right\} .
$$

It is straightforward to verify that the function $h(s)=(N / p)^{1 / s} s^{-1+\alpha}$ is nonincreasing when $\alpha \leq 1$ and attains its maximum in $s=\max \{2, p / N\}=2$ or in $s=p$ when $\alpha>1$. Therefore, when $N \geq p$,

$$
\left\|\sum_{i=1}^{N} z_{i}\right\|_{L_{p}} \leq c_{1} \kappa_{1} \max \left\{\sqrt{N p}, N^{1 / p} p^{\alpha}\right\}
$$


Finally, if $N \geq p^{2 \alpha-1}$ then $e^{2 \alpha-1} \sqrt{N p} \geq N^{1 / p} p^{\alpha}$, which completes the proof.

Proof of Theorem A. Consider $N \geq c_{1} s \log (e n / s) / \beta^{2}$. By Corollary 2.5, with probability at least $1-\exp \left(-c_{2} N \beta^{2}\right)$,

$$
\inf _{t \in \Sigma_{s} \cap S^{n-1}} \frac{1}{N} \sum_{i=1}^{N}\left\langle X_{i}, t\right\rangle^{2}>\frac{u^{2} \beta}{2} .
$$

Set $\left(X_{i}\right)_{i=1}^{N}$ for which (2.5) holds and let $\Gamma=N^{-1 / 2} \sum_{i=1}^{N}\left\langle X_{i}, \cdot\right\rangle f_{i}$. By Lemma 2.7 for $\lambda^{2}=u^{2} \beta / 2$, it follows that when $r \geq 1$,

$$
\inf _{t \in \sqrt{r} B_{1}^{n} \cap S^{n-1}}\|\Gamma t\|_{2}^{2} \geq \lambda^{2}-\frac{2 r}{s} \max _{1 \leq j \leq n}\left\|\Gamma e_{j}\right\|_{2}^{2} .
$$

Next, one has to obtain a high probability upper estimate on $\max _{1 \leq j \leq n}\left\|\Gamma e_{j}\right\|_{2}^{2}$. To that end, fix $w \geq 1$ and consider $z=x_{j}^{2}-1$ - where $x_{j}$ is the $j$-th coordinate of $X$. Observe that $z$ is a centered random variable and that $\|z\|_{L_{q}} \lesssim 4^{\alpha} \kappa_{1}^{2} q^{2 \alpha}$ for every $1 \leq q \leq \kappa_{2} \log (w n)$. Thus, by Lemma 2.8 for $p=\kappa_{2} \log (w n)$ and $c_{3}(\alpha) \sim 4^{\alpha} \exp ((4 \alpha-1))$,

$$
\left\|\frac{1}{N} \sum_{i=1}^{N} z_{i}\right\|_{L_{p}} \leq c_{3}(\alpha) \kappa_{1}^{2} \sqrt{\frac{p}{N}}
$$

provided that $N \geq p^{\max \{4 \alpha-1,1\}}=\left(\kappa_{2} \log (w n)\right)^{\max \{4 \alpha-1,1\}}$. Hence, if $N \geq$ $\left(c_{3}(\alpha) \kappa_{1}^{2}\right)^{2}\left(\kappa_{2} \log (w n)\right)^{\max \{4 \alpha-1,1\}}$, and setting $V_{j}=\left\|\Gamma e_{j}\right\|_{2}^{2}$, one has

$$
\left\|V_{j}\right\|_{L_{p}}=\left\|\frac{1}{N} \sum_{i=1}^{N} x_{i, j}^{2}\right\|_{L_{p}} \leq 1+c_{3}(\alpha) \kappa_{1}^{2} \sqrt{\frac{\kappa_{2} \log (w n)}{N}} \leq 2 ;
$$

thus,

$$
\begin{aligned}
P\left(\max _{1 \leq j \leq n} V_{j} \geq 2 e\right) & \leq \sum_{j=1}^{n} P\left(V_{j} \geq 2 e\right) \leq \sum_{j=1}^{n}\left(\frac{\left\|V_{j}\right\|_{L_{p}}}{2 e}\right)^{p} \\
& \leq n\left(\frac{1}{e}\right)^{p}=\frac{1}{w^{\kappa_{2}} n^{\kappa_{2}-1}}
\end{aligned}
$$

Combining the two estimates, if

$$
N \gtrsim \max \left\{s \log (e n / s),\left(c_{3}(\alpha) \kappa_{1}^{2}\right)^{2}\left(\kappa_{2} \log (w n)\right)^{\max \{4 \alpha-1,1\}}\right\}
$$


and $r \leq s \lambda^{2} / 8 e=s u^{2} \beta / 16 e$, then with probability at least $1-\exp \left(-c_{2} N \beta^{2}\right)-$ $1 /\left(w^{\kappa_{2}} n^{\kappa_{2}-1}\right)$

$$
\inf _{t \in \sqrt{r} B_{1}^{n} \cap S^{n-1}}\|\Gamma t\|_{2}^{2} \geq \lambda^{2}-\frac{4 e r}{s} \geq \lambda^{2} / 2
$$

Therefore, by Lemma 2.1, $\Gamma$ satisfies the exact reconstruction property for vectors that are $c_{4} u^{2} \beta s$-sparse, as claimed.

Proof of Theorem D: Since the argument is almost identical to the one used in the proof of the second part of Corollary 2.5. we will only sketch the details. Observe that if $\Gamma=N^{-1 / 2} \sum_{i=1}^{N}\left\langle X_{i}, \cdot\right\rangle f_{i}$ and $\operatorname{ker}(\Gamma) \cap \Sigma_{s}=\{0\}$, then for any $x_{0} \in \Sigma_{\lfloor s / 2\rfloor}$, the only $t \in \mathbb{R}^{n}$ for which $\Gamma t=\Gamma x_{0}$ and $\|t\|_{0} \leq\left\|x_{0}\right\|_{0}$ is $x_{0}$ itself. Thus, it suffices to show that for every $x \in \Sigma_{s} \cap S^{n-1},\left|\left\langle X_{i}, x\right\rangle\right|>0$ for some $1 \leq i \leq n$. Since $\Sigma_{s} \cap S^{n-1}$ is a union of $\left(\begin{array}{l}n \\ s\end{array}\right)$ spheres, the claim follows from Lemma 2.3 applied to each one of those spheres and for $u=0$, combined with a union bound argument.

\section{Proof of Theorem $\mathrm{C}$ and Theorem $\mathrm{C}^{\prime}$}

Consider an $N \times n$ matrix $\Gamma$ and $J \subset\{1, \ldots, n\}$. Set $\Gamma_{J}$ to be the $(N \times|J|)$ restriction of $\Gamma$ to $\operatorname{span}\left\{e_{j}: j \in J\right\}$. Recall that $B_{1}^{n}$ is the unit ball in $\ell_{1}^{n}=\left(\mathbb{R}^{n},\|\cdot\|_{1}\right)$, and put $B_{1}^{J^{c}}$ to be the set of vectors in $B_{1}^{n}$ that are supported in $J^{c}$ - the complement of $J$ in $\{1, \ldots, n\}$.

Lemma 3.1 Fix integers $s, N \leq n$ and $J \subset\{1, \ldots, n\}$ of cardinality at most s. If $v \in \mathbb{R}^{n}$ is supported in $J,\|v\|_{1}=1$ and $\Gamma v \in \Gamma B_{1}^{J^{c}}$, then $\Gamma$ does not satisfy the exact reconstruction property of order $s$.

Proof. Let $w \in B_{1}^{J^{c}}$ for which $\Gamma v=\Gamma w$ and observe that $v \neq w$ (otherwise, $v \in B_{1}^{J} \cap B_{1}^{J^{c}}$, implying that $v=0$, which is impossible because $\|v\|_{1}=1$ ).

Since $\|w\|_{1} \leq 1=\|v\|_{1}, w$ is at least as good a candidate as $v$ for the $\ell_{1}$-minimization problem $\min \left(\|t\|_{1}: \Gamma t=\Gamma v\right)$; hence, $v$ is not the unique solution of that problem.

Set $x_{\cdot 1}, \cdots, x_{\cdot n}$ to be the columns of $\Gamma$. It immediately follows from Lemma 3.1 that if one wishes to prove that $\Gamma$ does not satisfy $\operatorname{ER}(1)$, it suffices to show that, for instance the first basis vector $e_{1}$ cannot be exactly reconstruct. This follows from

$$
\Gamma e_{1}=x_{\cdot 1} \in \operatorname{absconv}\left(x_{\cdot k}: k \neq 1\right)=\operatorname{absconv}\left(\Gamma e_{k}: k \neq 1\right)=\Gamma B_{1}^{\{1\}^{c}},
$$


where $\operatorname{absconv}(S)$ is the convex hull of $S \cup-S$. Therefore, if

$$
\left\|x_{\cdot 1}\right\|_{2} \leq c_{0} \quad \text { and } \quad c_{0} B_{2}^{N} \subset \operatorname{absconv}\left(x_{\cdot k}: k \neq 1\right)
$$

for some absolute constant $c_{0}$, then $\Gamma$ does not satisfy $\operatorname{ER}(1)$.

The proofs of Theorem $\mathrm{C}$ and of Theorem $\mathrm{C}^{\prime}$ follow from the construction of a random matrix ensemble for which (3.1) holds with probability larger than $1 / 2$. We now turn on to such a construction.

Let $\eta$ be a selector (a $\{0,1\}$-valued random variable) with mean $\delta$ to be named later, and let $\varepsilon$ be a symmetric $\{-1,1\}$-valued random variable that is independent of $\eta$. Fix $R>0$ and set

$$
z=\varepsilon(1+R \eta)
$$

Observe that if $p \geq 2$ and $R \geq 1$ then

$$
\frac{\|z\|_{L_{p}}}{\|z\|_{L_{2}}}=\frac{\left(1+\left((1+R)^{p}-1\right) \delta\right)^{1 / p}}{\left(1+\left((1+R)^{2}-1\right) \delta\right)^{1 / 2}} \sim \frac{\left(1+R^{p} \delta\right)^{1 / p}}{\left(1+R^{2} \delta\right)^{1 / 2}} \sim R \delta^{1 / p}
$$

and the last equivalence holds when $R^{2} \delta \lesssim 1$ and $R^{p} \delta \gtrsim 1$. Fix $2<p \leq$ $2 \log (1 / \delta)$ which will be specified later and set $R=\sqrt{p}(1 / \delta)^{1 / p}$. Since the function $q \rightarrow \sqrt{q} / \delta^{1 / q}$ is decreasing for $2 \leq q \leq 2 \log (1 / \delta)$ one has that for $2 \leq q \leq p$ and for $\delta$ that is small enough,

$$
\|z\|_{L_{q}} \leq c_{0} \sqrt{q}\|z\|_{L_{2}}
$$

Note that $x=z /\|z\|_{L_{2}}$ is a mean-zero, variance one random variable that exhibits a 'subgaussian' moment behaviour only up to $p$. Indeed, if $2 \leq q \leq p,\|z\|_{L_{q}} \lesssim \sqrt{q}\|z\|_{L_{2}}$, and if $q>p,\|z\|_{L_{q}} \sim \sqrt{p} \delta^{1 / q-1 / p}\|z\|_{L_{2}}$, which may be far larger than $\sqrt{q}\|z\|_{L_{2}}$ if $\delta$ is sufficiently small.

Let $X=\left(x_{1}, \ldots, x_{n}\right)$ be a vector whose coordinates are independent, distributed as $x$ and let $\Gamma$ be the measurement matrix generated by $x$. Note that up to the normalization factor of $\|z\|_{L_{2}}$, which is of the order of a constant when $R^{2} \delta \lesssim 1, \sqrt{N} \Gamma$ is a perturbation of a Rademacher matrix by a sparse matrix with few random spikes that are either $R$ or $-R$.

As noted earlier, if for every $t \in \mathbb{R}^{n}$,

$$
\|\langle X, t\rangle\|_{L_{4}} \leq C\|\langle X, t\rangle\|_{L_{2}},
$$

then the small-ball condition holds with constants that depend only on $C$. To show that $X$ satisfies $(3.2)$, denote by $\mathbb{E}_{\eta}$ (resp. $\mathbb{E}_{\varepsilon}$ ) the expectation 
with respect to the $\eta$-variables (resp. $\varepsilon$-variables), and observe that by a straightforward application of Khintchine's inequality (see, e.g., p.91 in [27]), for every $t \in \mathbb{R}^{n}$,

$$
\begin{aligned}
& \mathbb{E}\langle X, t\rangle^{4} \lesssim \mathbb{E}_{\eta} \mathbb{E}_{\varepsilon}\left(\sum_{j=1}^{n} \varepsilon_{j}\left(1+R \eta_{j}\right) t_{j}\right)^{4} \lesssim \mathbb{E}_{\eta}\left(\sum_{j=1}^{n}\left(1+R \eta_{j}\right)^{2} t_{j}^{2}\right)^{2} \\
& =\mathbb{E}_{\eta} \sum_{k, \ell}\left(1+R \eta_{k}\right)^{2} t_{k}^{2}\left(1+R \eta_{\ell}\right)^{2} t_{\ell}^{2} \lesssim\|t\|_{2}^{4}=\left(\mathbb{E}\langle X, t\rangle^{2}\right)^{2},
\end{aligned}
$$

provided that $R^{4} \delta \lesssim 1$.

Set $\left(f_{i}\right)_{i=1}^{N}$ to be the canonical basis of $\mathbb{R}^{N}$ and put $\tilde{\Gamma}=\left(z_{\ell k}\right)=\|z\|_{L_{2}} \sqrt{N} \Gamma$, an $N \times n$ matrix whose entries are independent copies of $z$. Let

$$
v_{j}=\tilde{\Gamma} e_{j}=\sum_{\ell=1}^{N} z_{\ell j} f_{j},
$$

and consider

$$
V=\operatorname{absconv}\left(\left\{v_{j}: 2 \leq j \leq n\right\}\right),
$$

the convex hull of $\left( \pm v_{j}\right)_{j=2}^{n}$.

We will show that with probability at least $1 / 2, \sqrt{N} B_{2}^{N} \subset V$ and $\left\|v_{1}\right\|_{2} \leq$ $\sqrt{N}$, in three steps:

Lemma 3.2 With probability at least 3/4, for every $1 \leq i \leq N$ there is $y_{i} \in B_{\infty}^{N}$ for which $y_{i}+R f_{i} \in V$.

In other words, with non-trivial probability, $V$ contains a perturbation of all the vectors $R f_{i}, i=1, \ldots, N$, and thus, $V$ 'almost' contains $R B_{1}^{N}$.

Proof. Fix a realization of the $N \times n$ Rademacher matrix $\left(\varepsilon_{\ell j}\right)$ and note that for every $1 \leq i \leq N$ and every $2 \leq j \leq n$

$$
v_{j}=\sum_{\ell=1}^{N} \varepsilon_{\ell j} f_{\ell}+\varepsilon_{i j} R f_{i}
$$

if $\eta_{i j}=1$ and for every $\ell \neq i, \eta_{\ell j}=0$. Moreover, if this happens, and since $V$ is centrally symmetric (that is, if $v \in V$ then $-v \in V$ ),

$$
\varepsilon_{i j}\left(\sum_{\ell=1}^{N} \varepsilon_{\ell j} f_{\ell}\right)+R f_{i}=y_{i}+R f_{i} \in V,
$$


and $y_{i} \in B_{\infty}^{N}$.

Thus, it remains to estimate the probability that for every $1 \leq i \leq N$ there is some $2 \leq j \leq n$ for which $\eta_{i j}=1$ and for every $\ell \neq i, \eta_{\ell j}=0$. Clearly, for every $1 \leq i \leq N$,

$$
\begin{aligned}
& P_{\eta}\left(\text { there exists } j \in\{2, \ldots, n\}: \eta_{i j}=1, \text { and } \eta_{\ell j}=0 \text { if } \ell \neq i\right) \\
& =1-\left(1-(1-\delta)^{N-1} \delta\right)^{n-1} \geq 1-\frac{1}{4 N}
\end{aligned}
$$

provided that

$$
\frac{\log N}{n} \lesssim \delta \lesssim \frac{\log (e n / N)}{N}
$$

Hence, the claim follows by the union bound and integration with respect to the $\left(\varepsilon_{i j}\right)$.

Next, it is straightforward to verify that when $V$ contains such a perturbation of $R B_{1}^{N}$ (by vectors in $B_{\infty}^{N}$ ), it must also contain a large Euclidean ball, assuming that $R$ is large enough.

Lemma 3.3 Let $R>N$, and for every $1 \leq i \leq N$, set $y_{i} \in B_{\infty}^{N}$ and put $v_{i}=R f_{i}+y_{i}$. If $V$ is a convex, centrally symmetric set, and if $v_{i} \in V$ for every $1 \leq i \leq N$ then $(R / \sqrt{N}-\sqrt{N}) B_{2}^{N} \subset V$.

Proof. A separation argument shows that if $\sup _{v \in V}|\langle v, w\rangle| \geq \rho$ for every $w \in S^{N-1}$, then $\rho B_{2}^{N} \subset V$ (indeed, otherwise there would be some $x \in$ $\rho B_{2}^{N} \backslash V$; but it is impossible to separate $x$ and the convex and centrally symmetric $V$ using any norm-one functional).

To complete the proof, observe that for every $w \in S^{N-1}$,

$$
\begin{aligned}
& \sup _{v \in V}|\langle v, w\rangle| \geq \max _{1 \leq i \leq N}\left|\left\langle R f_{i}+y_{i}, w\right\rangle\right| \\
& \geq \max _{1 \leq i \leq N}\left|\left\langle R f_{i}, w\right\rangle\right|-\max _{1 \leq i \leq N}\left|\left\langle y_{i}, w\right\rangle\right| \geq R / \sqrt{N}-\sqrt{N} .
\end{aligned}
$$

Applying Lemma 3.3. it follows that if $R \geq 2 N$ then with probability at least $3 / 4, \sqrt{N} B_{2}^{N} \subset V$. Finally, if $\delta \lesssim 1 / N$ then

$$
P\left(\sum_{\ell=1}^{N} z_{\ell 1} f_{\ell} \in \sqrt{N} B_{2}^{N}\right) \geq P\left(\left\|\sum_{\ell=1}^{N} z_{\ell 1} f_{\ell}\right\|_{2}=\sqrt{N}\right)=(1-\delta)^{N} \geq 3 / 4 .
$$


Hence, with probability at least $1 / 2$,

$$
\sum_{\ell=1}^{N} z_{\ell 1} f_{\ell}=\tilde{\Gamma} e_{1} \in V=\operatorname{absconv}\left(\tilde{\Gamma} e_{j}: j \in\{2, \ldots, n\}\right),
$$

and the same assertion holds for the normalized matrix $\Gamma$, showing that it does not satisfy ER(1).

Of course, this assertion holds under several conditions on the parameters involved: namely, that $R=\sqrt{p}(1 / \delta)^{1 / p} \geq 2 N$; that $(\log N) / n \lesssim \delta \lesssim$ $\log (e n / N) / N$; that $R^{4} \delta \lesssim 1$; that $p \leq 2 \log (1 / \delta)$ and that $\delta \lesssim 1 / N$.

For instance, one may select $\delta \sim(\log N) / n$ and $p \sim(\log n) / \log N$, in which case all these conditions are met; hence, with probability at least $1 / 2$, $\Gamma$ does not satisfy $\mathrm{ER}(1)$, proving Theorem C. A similar calculation leads to the proof of Theorem $\mathrm{C}^{\prime}$.

Remark 3.4 Note that the construction leads to a stronger, non-uniform result, namely, that for every basis vector $e_{k}$, with probability at least $1 / 2, e_{k}$ is not the unique solution of $\min \left(\|t\|_{1}: \Gamma t=\Gamma e_{k}\right)$. In particular, uniformity over all supports of size 1 in the definition of ER(1) is not the reason why the moment assumption in Theorem $A$ is required.

\section{Results in the noisy measurements setup}

In previous sections, we considered the idealized scenario, in which the data was noiseless. Here, we will study the noisy setup: one observes $N$ couples $\left(z_{i}, X_{i}\right)_{i=1}^{N}$, and each $z_{i}$ is a noisy observation of $\left\langle X_{i}, x_{0}\right\rangle$ :

$$
z_{i}=\left\langle X_{i}, x_{0}\right\rangle+g_{i}, \quad i=1, \ldots, N .
$$

The goal is to obtain as much information as possible on the unknown vector $x_{0}$ with only the data $\left(z_{i}, X_{i}\right)_{i=1}^{N}$ at one's disposal, and for the sake of simplicity, we will assume that the $g_{i}$ 's are independent Gaussian random variables $\mathcal{N}\left(0, \sigma^{2}\right)$ that are also independent of the $X_{i}$ 's.

Unlike the noiseless case, there is no hope of reconstructing $x_{0}$ from the given data, and instead of exact reconstruction, there are three natural questions that one may consider:

- The estimation problem: given some norm $\|\cdot\|$ on $\mathbb{R}^{n}$, one would like to construct a procedure $\hat{x}$ for which $\left\|\hat{x}-x_{0}\right\|$ is as small as possible. 
- The prediction problem: given a new (random, independent) 'input' $X \in$ $\mathbb{R}^{n}$, one has to find a good guess $\langle\hat{x}, X\rangle$ of the most likely associated output $z$, knowing that $(z, X)$ shares the same distribution with the other couples $\left(z_{1}, X_{1}\right), \ldots,\left(z_{N}, X_{N}\right)$.

- The de-noising problem: given a norm $\|\cdot\|$ on $\mathbb{R}^{N}$ and a measurement matrix $\Gamma$, one has to construct $\hat{x}$ for which $\left\|\Gamma \hat{x}-\Gamma x_{0}\right\|$ is small.

These three problems are central in modern Statistics, and are featured in numerous statistical monographs, particularly in the context of the Gaussian regression model (Equation (4.1)).

Recently, all three problems have been recast in a 'high-dimensional' scenario, in which the number of observations $N$ may be much smaller than the ambient dimension $n$. Unfortunately, such problems are often impossible to solve without additional assumptions, and just as in the noiseless case, the situation improves dramatically if $x_{0}$ has some low-dimensional structure, for example, if it is $s$-sparse. The aim is therefore to design a procedure that performs as if the true dimension of the problem is $s$ rather than $n$, despite the noisy data.

To that end, $\ell_{0}$ penalization methods, sometimes called Model Selection procedures, have been introduced and studied extensively (see, e.g., [29, 4] for results in the context of the model (4.1), as well as in other examples). However, just as in the noise-free problem, the obvious downside of $\ell_{0}$ penalization methods is that they are not feasible computationally. This has lead to the introduction of convex relaxations, based on $\ell_{1}$ minimization.

Two well established $\ell_{1}$-based procedures are the $L A S S O$ (see, e.g., [43]) defined by

$$
\hat{x}_{\lambda} \in \underset{x \in \mathbb{R}^{n}}{\operatorname{argmin}}\left(\frac{1}{N} \sum_{i=1}^{N}\left(z_{i}-\left\langle X_{i}, x\right\rangle\right)^{2}+\lambda\|x\|_{1}\right),
$$

and the Dantzig selector (see [1]).

Both procedures may be implemented effectively, and their estimation and de-noising properties have been obtained under some assumptions on the measurement matrix (see, e.g. 6, 3, 44] or Chapters 7 and 8 in [24]).

In this section, we shall focus on two such conditions on the measurement matrix. The first, called the Compatibility Condition, was introduced in 44] (see also Definition 2.1 in [45]); the second, the Restricted Eigenvalue Condition, was introduced in [3]. 
Definition 4.1 Let $\Gamma$ be an $N \times n$ matrix. For $L>0$ and a set $S \subset$ $\{1, \ldots, n\}$, the compatibility constant associated with $L$ and $S$ is

$$
\phi(L, S)=\sqrt{|S|} \min \left(\left\|\Gamma \zeta_{S}-\Gamma \zeta_{S^{c}}\right\|_{2}:\left\|\zeta_{S}\right\|_{1}=1,\left\|\zeta_{S^{c}}\right\|_{1} \leq L\right),
$$

where $\zeta_{S}\left(\right.$ resp. $\left.\zeta_{S^{c}}\right)$ denotes a vector that is supported in $S$ (resp. $\left.S^{c}\right)$.

$\Gamma$ satisfies the Compatibility Condition for the set $S_{0}$ with constants $L>1$ and $c_{0}$ if $\phi\left(L, S_{0}\right) \geq c_{0}$; it satisfies the uniform Compatibility Condition $(C C)$ of order $s$ if $\min _{|S| \leq s} \phi(L, S) \geq c_{0}$.

A typical result for the LASSO in the Gaussian model (4.1) and when $\Gamma$ satisfies the Compatibility Condition, is Theorem 6.1 in [6]:

Theorem 4.2 ([6], Theorem 6.1) Let $x_{0} \in \mathbb{R}^{n}$ be some fixed vector and assume that the data $\left(z_{i}, X_{i}\right)_{i=1}^{N}$ have been drawn according to the Gaussian regression model (4.1). Denote by $\Gamma=N^{-1 / 2} \sum_{i=1}^{N}\left\langle X_{i}, \cdot\right\rangle f_{i}$ the measurement matrix. Let $t>0$. If $S_{0}$ is the support of $x_{0}$ and $\lambda=4 \sigma \sqrt{\left(t^{2}+\log n\right) / N}$, then with probability larger than $1-2 \exp \left(-t^{2} / 2\right)$,

$\left\|\Gamma \hat{x}_{\lambda}-\Gamma x_{0}\right\|_{2}^{2} \leq \frac{64 \sigma^{2}\left\|x_{0}\right\|_{0}\left(t^{2}+\log n\right)}{N \phi^{2}\left(3, S_{0}\right)}$ and $\left\|\hat{x}_{\lambda}-x_{0}\right\|_{1} \leq \frac{64 \sigma\left\|x_{0}\right\|_{0}}{\phi^{2}\left(3, S_{0}\right)} \sqrt{\frac{t^{2}+\log n}{N}}$.

Even though the Compatibility Condition in $S_{0}$ suffices to show that the LASSO is an effective procedure, the fact remains that $S_{0}$ is not known. And while a non-uniform approach is still possible (e.g., if $\Gamma$ is a random matrix, one may try showing that with high probability it satisfies the Compatibility Condition for the fixed, but unknown $S_{0}$ ), the uniform Compatibility Condition is a safer requirement - and the one we shall explore below.

Another uniform condition of a similar flavour is the Restricted Eigenvalue Condition from [3]. To define it, let us introduce the following notation: for $x \in \mathbb{R}^{n}$ and a set $S_{0} \subset\{1, \ldots, n\}$ of cardinality $\left|S_{0}\right| \leq s$, let $S_{1}$ be the subset of indices of the $m$ largest coordinates of $\left(\left|x_{i}\right|\right)_{i=1}^{n}$ that are outside $S_{0}$. Let $x_{S_{01}}$ be the restriction of $x$ to the set $S_{01}=S_{0} \cup S_{1}$.

Definition 4.3 Let $\Gamma$ be an $N \times n$ matrix. Given $c_{0} \geq 1$ and an integer $1 \leq s \leq m \leq n$ for which $m+s \leq n$, the restricted eigenvalue constant is

$\kappa\left(s, m, c_{0}\right)=\min \left(\frac{\|\Gamma x\|_{2}}{\left\|x_{S_{01}}\right\|_{2}}: S_{0} \subset\{1, \ldots, n\},\left|S_{0}\right| \leq s,\left\|x_{S_{0}^{c}}\right\|_{1} \leq c_{0}\left\|x_{S_{0}}\right\|_{1}\right)$.

The matrix $\Gamma$ satisfies the Restricted Eigenvalue Condition (REC) of order $s$ with a constant $c$ if $\kappa(s, s, 3) \geq c$. 
Estimation and de-noising results follow from Theorem 6.1 (for the Dantzig selector) and Theorem 6.2 (for the LASSO) in 3, when the measurement matrix $\Gamma$, normalized by having the diagonal elements of $\Gamma^{\top} \Gamma$ equal 1 , satisfies the REC of an appropriate order and with a constant that is independent of the dimension. We also refer to Lemma 6.10 in [6] for similar results that do not require normalization.

Because the two lead to bounds on the performance of the LASSO and the Dantzig selector, a question that comes to mind is whether there are matrices that satisfy the CC or the REC. And, as in Compressed Sensing, the only matrices that are known to satisfy those conditions for the optimal number of measurements (rows) are well-behaved random matrices (see [35, 39, 34, 46] for some examples).

Our aim in this final section is to extend our results to the noisy setup, by identifying almost necessary and sufficient moment assumptions for the $\mathrm{CC}$ and the REC. This turns out to be straightforward: on one hand, the proof of Theorem A actually provides a stronger quantitative version of the exact reconstruction property; on the other, the uniform compatibility condition can be viewed as a quantitative version of a geometric condition on the polytope $\Gamma B_{1}^{n}$ that characterizes Exact Reconstruction. A similar observation is true for the REC: it can be viewed as a quantitative version of the null space property (see [18, 19] and below) which is also equivalent to the exact reconstruction property.

Definition 4.4 Let $1 \leq s \leq N$. A centrally symmetric polytope $P \subset \mathbb{R}^{N}$ is $s$-neighbourly if every set of $s$ of its vertices, containing no antipodal pair, is the set of all vertices of some face of $P$.

It is well known [16] that $\Gamma$ satisfies $\operatorname{ER}(s)$ if and only if $\Gamma B_{1}^{n}$ has $2 n$ vertices and $\Gamma B_{1}^{n}$ is a centrally symmetric $s$-neighbourly polytope. It turns out that this property is characterized by the uniform CC.

Lemma 4.5 Let $\Gamma$ be an $N \times n$ matrix. The following are equivalent:

1. $\Gamma B_{1}^{n}$ has $2 n$ vertices and is s-neighbourly,

2. $\min (\phi(1, S): S \subset\{1, \ldots, n\},|S| \leq s)>0$.

In particular, $\min _{|S| \leq s} \phi(L, S)$ for some $L \geq 1$ is a quantitative measure of the $s$-neighbourly property of $\Gamma B_{1}^{n}$ : if $\Gamma B_{1}^{n}$ is $s$-neighbourly and has $2 n$ vertices then the two sets

$$
\left\{\Gamma \zeta_{S}:\left\|\zeta_{S}\right\|_{1}=1\right\} \text { and }\left\{\Gamma \zeta_{S^{c}}:\left\|\zeta_{S^{c}}\right\|_{1} \leq 1\right\}
$$


are disjoint for every $|S| \leq s$. However, $\min _{|S| \leq s} \phi(1, S)$ measures how far the two sets are from one another, uniformly over all subsets $S \subset\{1, \ldots, n\}$ of cardinality at most $s$.

Proof. Let $C_{1}, \ldots, C_{n}$ be the $n$ columns of $\Gamma$. It follows from Proposition 2.2.13 and Proposition 2.2.16 in [12] that $\Gamma B_{1}^{n}$ has $2 n$ vertices and is a centrally symmetric $s$-neighbourly polytope if and only if for every $S \subset$ $\{1, \ldots, n\}$ of cardinality $|S| \leq s$ and every choice of signs $\left(\varepsilon_{i}\right) \in\{-1,1\}^{S}$,

$$
\operatorname{conv}\left(\left\{\varepsilon_{i} C_{i}: i \in S\right\}\right) \cap \operatorname{absconv}\left(\left\{C_{j}: j \notin S\right\}\right)=\emptyset .
$$

It is straightforward to verify that

$$
\bigcup_{\left(\varepsilon_{i}\right) \in\{ \pm 1\}^{S}} \operatorname{conv}\left(\left\{\varepsilon_{i} C_{i}: i \in S\right\}\right)=\left\{\Gamma \zeta_{S}:\left\|\zeta_{S}\right\|_{1}=1\right\}
$$

and that

$$
\operatorname{absconv}\left(\left\{C_{j}: j \notin S\right\}\right)=\left\{\Gamma \zeta_{S^{c}}:\left\|\zeta_{S^{c}}\right\|_{1} \leq 1\right\} .
$$

As a consequence, (4.5) holds for every $S \subset\{1, \ldots, n\}$ of cardinality at most $s$ if and only if $\min (\phi(1, S): S \subset\{1, \ldots, n\},|S| \leq s)>0$.

An observation of a similar nature is true for the REC: it can be viewed as a quantitative measure of the null space property.

Definition 4.6 Let $\Gamma$ be an $N \times n$ matrix. $\Gamma$ satisfies the null space property of order $s$ if it is invertible in the cone

$$
\left\{x \in \mathbb{R}^{n}: \text { there exists } S \subset\{1, \ldots, n\},|S| \leq s \text { and }\left\|x_{S^{c}}\right\|_{1} \leq\left\|x_{S}\right\|_{1}\right\} .
$$

In [18, 19, the authors prove that $\Gamma$ satisfies $\operatorname{ER}(s)$ if and only if it has the null space property of order $s$.

A natural way of quantifying the invertibility of $\Gamma$ in the cone (4.6) is to consider its smallest singular value, restricted to this cone, which is simply the REC $\kappa(s, n-s, 1)$. Unfortunately, statistical properties of the LASSO and of the Dantzig selector are not known under the assumption that $\kappa(s, n-s, 1)$ is an absolute constant (though if $\kappa(s, s, 3)$ is an absolute constant, LASSO is known to be optimal [3]).

The main result of this section is the following:

Theorem E. Let $L>0,1 \leq s \leq n$ and $c_{0}>0$. Under the same assumptions as in Theorem $A$ and with the same probability estimate, $\Gamma=$ $N^{-1 / 2} \sum_{i=1}^{N}\left\langle X_{i}, \cdot\right\rangle f_{i}$ satisfies: 
1. A uniform compatibility condition of order $c_{1} s$, namely that

$$
\min _{|S| \leq c_{1} s} \phi(L, S) \geq u^{2} \beta / 4
$$

$$
\text { for } c_{1}=u^{2} \beta /\left(16 e(1+L)^{2}\right) \text {. }
$$

2. A restricted eigenvalue condition of order $c_{2} s$, with

$$
\kappa\left(c_{2} s, m, c_{0}\right) \geq u^{2} \beta / 4
$$

for any $1 \leq m \leq n$, as long as $\left(1+c_{0}\right)^{2} c_{2} \leq u^{2} \beta /(16 e)$.

On the other hand, if $\Gamma$ is the matrix considered in Theorem $C$, then with probability at least $1 / 2, \phi\left(1,\left\{e_{1}\right\}\right)=0$ and $\kappa(1, m, 1)=0$ for any $1 \leq m \leq n$.

Just like Theorem A and Theorem C, Theorem E shows that the requirement that the coordinates of the measurement vector have $\log n$ moments is almost a necessary and sufficient condition for the uniform Compatibility Condition and the Restricted Eigenvalue Condition to hold. Moreover, it shows the significance of the small-ball condition, even in the noisy setup.

It also follows from Theorem $\mathrm{E}$ that if $X$ satisfies the small-ball condition and its coordinates have $\log n$ well-behaved moments as in Theorem $\mathrm{A}$, then $\Gamma B_{1}^{n}$ has $2 n$ vertices and is $s$-neighbourly with high probability for $N \sim$ $s \log (e n / s)$. In particular, this improves Theorem 4.3 in [1] by a logarithmic factor for matrices generated by subexponential variables.

Proof of Theorem E: Fix a constant $c_{1}$ to be named later and let $S \subset\{1, \ldots, n\}$ of cardinality $|S| \leq c_{1} s$. Let $\zeta_{S} \in \mathbb{R}^{n}$ be a vector supported on $S$ with $\left\|\zeta_{S}\right\|_{1}=1$ and let $\zeta_{S^{c}} \in \mathbb{R}^{n}$ be supported on $S^{c}$ with $\left\|\zeta_{S^{c}}\right\|_{1} \leq L$.

Consider $\gamma=\left(\zeta_{S}-\zeta_{S^{c}}\right) /\left\|\zeta_{S}-\zeta_{S^{c}}\right\|_{2}$. Since

$$
\left\|\zeta_{S}-\zeta_{S^{c}}\right\|_{2} \geq\left\|\zeta_{S}\right\|_{2} \geq \frac{\left\|\zeta_{S}\right\|_{1}}{\sqrt{|S|}}=\frac{1}{\sqrt{|S|}}
$$

it follows that $\gamma \in((1+L) \sqrt{|S|}) B_{1}^{n} \cap S^{n-1}$.

Recall that by (2.7), if $r=(1+L)^{2} c_{1} s \leq s u^{2} \beta /(16 e)$, then $\|\Gamma \gamma\|_{2} \geq$ $\left(u^{2} \beta\right) / 4$. Therefore,

$$
\left\|\Gamma \zeta_{S}-\Gamma \zeta_{S^{c}}\right\|_{2} \geq \frac{u^{2} \beta}{4}\left\|\zeta_{S}-\zeta_{S^{c}}\right\|_{2} \geq \frac{u^{2} \beta}{4}\left\|\zeta_{S}\right\|_{2} \geq \frac{u^{2} \beta\left\|\zeta_{S}\right\|_{1}}{4 \sqrt{|S|}}=\frac{u^{2} \beta}{4 \sqrt{|S|}}
$$

and thus $\min _{|S| \leq c_{1} s} \phi(L, S) \geq u^{2} \beta / 4$ for $c_{1}=u^{2} \beta /\left(16 e(1+L)^{2}\right)$. 
Turning to the REC, fix a constant $c_{2}$ to be named later. Consider $x$ in the cone and let $S_{0} \subset\{1, \ldots, n\}$ of cardinality $\left|S_{0}\right| \leq c_{2} s$ for which $\left\|x_{S_{0}^{c}}\right\|_{1} \leq c_{0}\left\|x_{S_{0}}\right\|_{1}$. Let $S_{1} \subset\{1, \ldots, n\}$ be the set of indices of the $m$ largest coordinates of $\left(\left|x_{i}\right|\right)_{i=1}^{n}$ that are outside $S_{0}$ and put $S_{01}=S_{0} \cup S_{1}$.

Observe that $\|x\|_{1} \leq\left(1+c_{0}\right)\left\|x_{S_{0}}\right\|_{1} \leq\left(1+c_{0}\right) \sqrt{\left|S_{0}\right|}\|x\|_{2}$; hence $x /\|x\|_{2} \in$ $\left(\left(1+c_{0}\right) \sqrt{\left|S_{0}\right|}\right) B_{1}^{n} \cap S^{n-1}$. Applying (2.7) again, if $\left(1+c_{0}\right)^{2} c_{2} s \leq s u^{2} \beta /(16 e)$, then $\|\Gamma x\|_{2} \geq\left(\left(u^{2} \beta\right) / 4\right)\|x\|_{2}$. Thus,

$$
\frac{\|\Gamma x\|_{2}}{\left\|x_{S_{01}}\right\|_{2}} \geq \frac{\|\Gamma x\|_{2}}{\|x\|_{2}} \geq \frac{u^{2} \beta}{4}
$$

and $\kappa\left(c_{2} s, m, c_{0}\right) \geq u^{2} \beta / 4$ for any $1 \leq m \leq n$, as long as $\left(1+c_{0}\right)^{2} c_{2} \leq$ $u^{2} \beta /(16 e)$.

The proof of the second part of Theorem E is an immediate corollary of the construction used in Theorem C. Recall that with probability at least $1 / 2, \Gamma e_{1} \in \operatorname{absconv}\left(\Gamma e_{j}: j \in\{2, \ldots, n\}\right)$. Setting $J=\left\{e_{2}, \ldots, e_{n}\right\}$, there is $\zeta \in B_{1}^{J}$ for which $\left\|\Gamma e_{1}-\Gamma \zeta\right\|_{2}=0$. Therefore, $\phi\left(1,\left\{e_{1}\right\}\right)=0$ and $\kappa(1, m, 1)=0$ for any $1 \leq m \leq n$, as claimed.

Remark 4.7 The results obtained in Theorem $A$ and in parts (1) and (2) of Theorem $E$ are also valid for the normalized (columns wise) measurement matrix:

$$
\Gamma_{1}=\Gamma \tilde{D}^{-1} \text { where } \tilde{D}=\operatorname{diag}\left(\left\|\Gamma e_{1}\right\|_{2}, \ldots,\left\|\Gamma e_{n}\right\|_{2}\right) .
$$

The proof is almost identical to the one used for $\Gamma$ itself, even though $\Gamma_{1}$ does not have independent rows vectors, due to the normalization. For the sake of brevity, we will not present the straightforward proof of this observation.

Finally, the counterexample constructed in the proof of Theorem $C$ and in which a typical $\Gamma$ does not satisfy $E R(1)$, does not necessarily generate $\Gamma B_{1}^{n}$ that is not s-neighbourly. Indeed, an inspection of the construction shows that the reason ER(1) fails is that $\Gamma B_{1}^{n}$ has less than $2 n-2$ vertices, rather than that $\Gamma B_{1}^{n}$ is not s-neighbourly. Thus, the question of whether a moment condition is necessary for the random polytope $\Gamma B_{1}^{n}$ to be s-neighbourly with probability at least $1 / 2$ is still unresolved.

\section{References}

[1] Radosław Adamczak, Alexander E. Litvak, Alain Pajor, and Nicole TomczakJaegermann. Restricted isometry property of matrices with independent columns and neighborly polytopes by random sampling. Constr. Approx., 34(1):61-88, 2011.

[2] Keith Ball. Cube slicing in $\mathbf{R}^{n}$. Proc. Amer. Math. Soc., 97(3):465-473, 1986. 
[3] Peter J. Bickel, Ya'acov Ritov, and Alexandre B. Tsybakov. Simultaneous analysis of LASSO and Dantzig selector. Ann. Statist., 37(4):1705-1732, 2009.

[4] Lucien Birgé and Pascal Massart. Gaussian model selection. J. Eur. Math. Soc. (JEMS), 3(3):203-268, 2001.

[5] Stéphane Boucheron, Gábor Lugosi, and Pascal Massart. Concentration Inequalities: A Nonasymptotic Theory of Independence. Oxford University Press, 2013. ISBN 978-0-19-953525-5.

[6] Peter Bühlmann and Sara A. van de Geer. Statistics for high-dimensional data. Springer Series in Statistics. Springer, Heidelberg, 2011. Methods, theory and applications.

[7] Emmanuel J. Candès. The restricted isometry property and its implications for compressed sensing. C. R. Math. Acad. Sci. Paris, 346(9-10):589-592, 2008.

[8] Emmanuel J. Candès, Justin Romberg, and Terence Tao. Robust uncertainty principles: exact signal reconstruction from highly incomplete frequency information. IEEE Trans. Inform. Theory, 52(2):489-509, 2006.

[9] Emmanuel J. Candès, Justin K. Romberg, and Terence Tao. Stable signal recovery from incomplete and inaccurate measurements. Comm. Pure Appl. Math., 59(8):1207-1223, 2006.

[10] Emmanuel J. Candès and Terence Tao. Near-optimal signal recovery from random projections: universal encoding strategies? IEEE Trans. Inform. Theory, 52(12):5406-5425, 2006.

[11] Emmanuel J. Candès and Terence Tao. The Dantzig selector: statistical estimation when $p$ is much larger than $n$. Ann. Statist., 35(6):2313-2351, 2007.

[12] Djalil Chafaï, Olivier Guédon, Guillaume Lecué, and Alain Pajor. Interactions between compressed sensing random matrices and high dimensional geometry, volume 37 of Panoramas et Synthèses [Panoramas and Syntheses]. Société Mathématique de France, Paris, 2012.

[13] Scott Shaobing Chen, David L. Donoho, and Michael A. Saunders. Atomic decomposition by basis pursuit. SIAM Rev., 43(1):129-159, 2001. Reprinted from SIAM J. Sci. Comput. 20 (1998), no. 1, 33-61 (electronic) [ MR1639094 (99h:94013)].

[14] Jon F. Claerbout and Francis Muir. Robust modeling with erratic data. Geophysics, 38(5):826-844, 1973.

[15] Víctor H. de la Peña and Evarist Giné. Decoupling. Probability and its Applications (New York). Springer-Verlag, New York, 1999. From dependence to independence, Randomly stopped processes. $U$-statistics and processes. Martingales and beyond.

[16] David L. Donoho. Neighborly polytopes and sparse solutions of under-determined linear equations. Technical report, Department of Statistics, Standford University, 2005.

[17] David L. Donoho. Compressed sensing. IEEE Trans. Inform. Theory, 52(4):12891306, 2006.

[18] David L. Donoho and Michael Elad. Optimally sparse representation in general (nonorthogonal) dictionaries via $l^{1}$ minimization. Proc. Natl. Acad. Sci. USA, 100(5):2197-2202 (electronic), 2003. 
[19] David L. Donoho and Xiaoming Huo. Uncertainty principles and ideal atomic decomposition. IEEE Trans. Inform. Theory, 47(7):2845-2862, 2001.

[20] David L. Donoho and Ben F. Logan. Signal recovery and the large sieve. SIAM J. Appl. Math., 52(2):577-591, 1992.

[21] R. M. Dudley. Central limit theorems for empirical measures. Ann. Probab., 6(6):899929 (1979), 1978.

[22] Simon Foucart. Stability and robustness of $\ell_{1}$-minimizations with Weibull matrices and redundant dictionaries. Linear Algebra Appl., 441:4-21, 2014.

[23] Simon Foucart and Holger Rauhut. A mathematical introduction to compressive sensing. Applied and Numerical Harmonic Analysis. Birkhäuser/Springer, New York, 2013.

[24] Vladimir Koltchinskii. Oracle inequalities in empirical risk minimization and sparse recovery problems, volume 2033 of Lecture Notes in Mathematics. Springer, Heidelberg, 2011. Lectures from the 38th Probability Summer School held in Saint-Flour, 2008, École d'Été de Probabilités de Saint-Flour. [Saint-Flour Probability Summer School].

[25] Vladimir Koltchinskii and Shahar Mendelson. Bounding the smallest singular value of a random matrix without concentration. Technical report, Technion and Georgia Tech, 2013. arXiv:1312.3580.

[26] Rafał Latała. Estimation of moments of sums of independent real random variables. Ann. Probab., 25(3):1502-1513, 1997.

[27] Michel Ledoux and Michel Talagrand. Probability in Banach spaces, volume 23 of Ergebnisse der Mathematik und ihrer Grenzgebiete (3) [Results in Mathematics and Related Areas (3)]. Springer-Verlag, Berlin, 1991. Isoperimetry and processes.

[28] Ben Logan. Properties of High-Pass Signals. PhD thesis, Columbia University, New York, 1965.

[29] Pascal Massart. Concentration inequalities and model selection, volume 1896 of Lecture Notes in Mathematics. Springer, Berlin, 2007. Lectures from the 33rd Summer School on Probability Theory held in Saint-Flour, July 6-23, 2003, With a foreword by Jean Picard.

[30] Shahar Mendelson. Learning without concentration. Journal of the ACM. To appear.

[31] Shahar Mendelson. A remark on the diameter of random sections of convex bodies. Geometric Aspects of Functional Analysis (GAFA Seminar Notes). Lecture notes in Mathematics 2116, pages 395-404, 2014.

[32] Shahar Mendelson, Alain Pajor, and Nicole Tomczak-Jaegermann. Uniform uncertainty principle for Bernoulli and subgaussian ensembles. Constr. Approx., 28(3):277289, 2008.

[33] B. K. Natarajan. Sparse approximate solutions to linear systems. SIAM J. Comput., 24(2):227-234, 1995.

[34] Roberto Imbuzeiro Oliveira. The lower tail of random quadratic forms, with applications to ordinary least squares and restricted eigenvalue properties. Technical report, IMPA, 2013. arXiv:1312.2903.

[35] Garvesh Raskutti, Martin J. Wainwright, and Bin Yu. Restricted eigenvalue properties for correlated Gaussian designs. J. Mach. Learn. Res., 11:2241-2259, 2010. 
[36] B. A. Rogozin. An estimate for the maximum of the convolution of bounded densities. Teor. Veroyatnost. i Primenen., 32(1):53-61, 1987.

[37] Mark Rudelson and Roman Vershynin. On sparse reconstruction from Fourier and Gaussian measurements. Comm. Pure Appl. Math., 61(8):1025-1045, 2008.

[38] Mark Rudelson and Roman Vershynin. Small ball probabilities for linear images of high dimensional distributions. Technical report, University of Michigan, 2013.

[39] Mark Rudelson and Shuheng Zhou. Reconstruction from anisotropic random measurements. IEEE Trans. Inform. Theory, 59(6):3434-3447, 2013.

[40] Fadil Santosa and William W. Symes. Linear inversion of band-limited reflection seismograms. SIAM J. Sci. Statist. Comput., 7(4):1307-1330, 1986.

[41] Nikhil Srivastava and Roman Vershynin. Covariance estimation for distributions with $2+\varepsilon$ moments. Ann. Probab., 41(5):3081-3111, 2013.

[42] Howard L. Taylor, Stephen C. Banks, and John F. McCoy. Deconvolution with the 11-norm. Geophysics, 44(1):39-52, 1979.

[43] Robert Tibshirani. Regression shrinkage and selection via the lasso. J. Roy. Statist. Soc. Ser. B, 58(1):267-288, 1996.

[44] Sara A. van de Geer. The deterministic lasso. In JSM proceedings. American Statistical Association, 140, 2007.

[45] Sara A. van de Geer. Weakly decomposable regularization penalties and structured sparsity. Technical report, ETH Zürich, 2013.

[46] Sara A. van de Geer and Alan Muro. On higher order isotropy conditions and lower bounds for sparse quadratic forms. Technical report, ETH Zürich, 2014.

[47] Aad W. van der Vaart and Jon A. Wellner. Weak convergence and empirical processes. Springer Series in Statistics. Springer-Verlag, New York, 1996. With applications to statistics. 